\title{
Significance of Self-Trapping on Hydrogen Diffusion
}

\section{Andreas Blomqvist, Gunnar K. Pálsson, C. Moysés Araújo, Rajeev Ahuja, and Björgvin Hjörvarsson \\ Department of Physics and Astronomy, Uppsala University, Box 516, S-751 20 Uppsala, Sweden}

(Received 17 May 2010; revised manuscript received 21 June 2010; published 28 October 2010)

The diffusion rate of hydrogen in $\mathrm{Nb}$ was calculated using ab initio molecular dynamics simulations. At low temperatures the hydrogen is strongly trapped in a local strain field which is caused by the elastic response of the lattice. At elevated temperatures, the residence time $(\tau)$ of hydrogen in an interstitial site is not sufficient for fully developing the local strain field. This unbinding of the interstitial hydrogen and the strain field increases the hopping rate $(1 / \tau)$ at elevated temperatures $(>400 \mathrm{~K})$. These results call for a revision of the conceptual framework of diffusion of hydrogen in transition metals at elevated temperatures.

DOI: 10.1103/PhysRevLett.105.185901

Metal-hydrogen systems serve as a prototype for lattice gas models. Because of the simplicity of the resulting changes in the electronic structure, hydrogen in metals can also be viewed as the most simple obtainable class of alloys. In the classical limit, where quantum effects can be ignored, the diffusion rate of hydrogen is experimentally well established for most metals [1-4]. At elevated temperatures, the diffusion rate is generally found to obey the phenomenological Arrhenius law and the underlying mechanism is commonly understood as interstitial hydrogen hopping in a static potential. The attempted jump rate is ascribed to the vibrational frequency of the interstitial, while the activation energy is thought to arise from the barrier separating the interstitial sites. This classical approach is conceptually appealing and describes the diffusion of hydrogen well at elevated temperatures [3].

When a hydrogen atom is absorbed in a transition metal, it attracts and localizes some of the neighboring metal $d$ electrons and as a consequence the interatomic metal binding diminishes $[5,6]$. The interstitial hydrogen thereby gives rise to a local strain field, which decays as $r^{-2}$ where $r$ is the distance from the hydrogen atom [3]. The displacement of the lattice in response to the interstitial hydrogen causes large changes in the energy landscape, resulting from a competition between a lowering of the total energy of the hydrogen and the repulsive elastic energy of the lattice. The equilibrium state of the displacement is called the self-trapped state and has been determined theoretically, using empirical potentials, in niobium by Fukai to $0.475 \mathrm{eV}$ [5], and by $\mathrm{Li}$ and Wahnström to $0.30 \mathrm{eV}$ [7]. Using ab initio methods, Sundell and Wahnström calculated the self-trapping energy to $0.189 \mathrm{eV}[8,9]$. The quantum theory of diffusion developed by Flynn and Stoneham [10] showed that the self-trapping has a big influence on tunneling. The effect of self-trapping on the energy landscape is illustrated in Fig. 1 and will be discussed later.

The interstitial hydrogen and its associated displacement field can be regarded as a quasiparticle and is often referred to as the small-polaron, or lattice polaron, in analogy with
PACS numbers: 66.30.jp, 66.30.Fq, 71.15.Mb, 71.15.Pd

the electron polaron in dielectric materials [11-13]. The polaron has an effective mass that depends on the displaced metal atoms and is typically much larger than the mass of the interstitial hydrogen. The diffusion of hydrogen in metals therefore involves two entities: the light interstitial and the strain field. Thus, although the Arrhenius description can be used to reproduce diffusion rates at elevated temperatures, the conceptual basis for it can be said to be incomplete. We will address this issue using $a b$ initio molecular dynamic simulations, including the diffusion of both the interstitial and the corresponding local strain field. The properties of hydrogen in niobium are

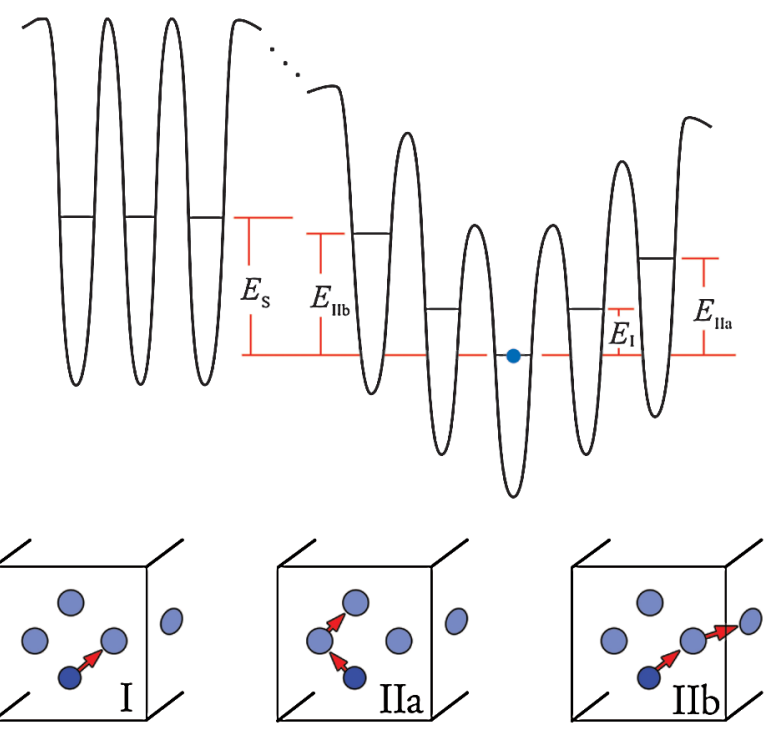

FIG. 1 (color online). The self-trapping lowers the hydrogenpolaron quasiparticle energy level $E_{S}$ with respect to the unstrained lattice. $E_{\mathrm{I}}$ is the difference in energy when moving the hydrogen to the closest tetrahedral site, keeping the position of the strain field fixed with respect to the lattice. $E_{\mathrm{IIa}}$ and $E_{\mathrm{IIb}}$ are similarly the energies for moving the hydrogen to the next nearest tetrahedral sites. The geometries of the jumps are shown in the bottom panel. 
exceedingly well explored and we will therefore use these results as a benchmark for our calculations.

The calculations were performed with one hydrogen atom in a supercell of $54 \mathrm{Nb}$ atoms, in the temperature range 200 to $2400 \mathrm{~K}$, using ab initio molecular dynamics (MD). The simulations were performed in steps of $50 \mathrm{~K}$ for temperatures below $1500 \mathrm{~K}$ and in steps of $100 \mathrm{~K}$ in the temperature range between 1500 and $2400 \mathrm{~K}$. At temperatures below $400 \mathrm{~K}$, the simulation times were too short to obtain good enough diffusion statistics. Forces were calculated from density functional theory [14] as implemented in the VASP code $[15,16]$. The ab initio calculations were based on the generalized gradient approximation [17] (GGA) and the projector-augmented wave (PAW) method [18]. A cutoff energy of $200 \mathrm{eV}$ was used. The $\Gamma$ point alone was sufficient for sampling the Brillouin zone. To check the k-point convergence on the diffusion, a $2 \times 2 \times 2$ Monkhorst-pack grid was used for an evaluation at $900 \mathrm{~K}$. The simulations were performed using a canonical ensemble with volume, particle number, and temperature fixed. The velocities were rescaled after every time step, with one time step corresponding to 1 femtosecond (fs). A simulation at $950 \mathrm{~K}$ using a microcanonical ensemble, with volume, particle number, and energy fixed, was used to validate the choice of ensemble. The simulation time for each temperature ranged from $200 \mathrm{ps}$ to $1 \mathrm{~ns}$, depending on temperature.

In the dilute $\alpha$ phase, hydrogen occupies tetrahedral sites in a random configuration [2,3] which causes an isotropic volume expansion [1]. Figure 2 shows an isosurface of the hydrogen density obtained from the simulations at $550 \mathrm{~K}$ where the hydrogen density clearly concentrates at the tetrahedral interstitial sites in the $\mathrm{Nb}$ bcc lattice, in good agreement with the experimental results.

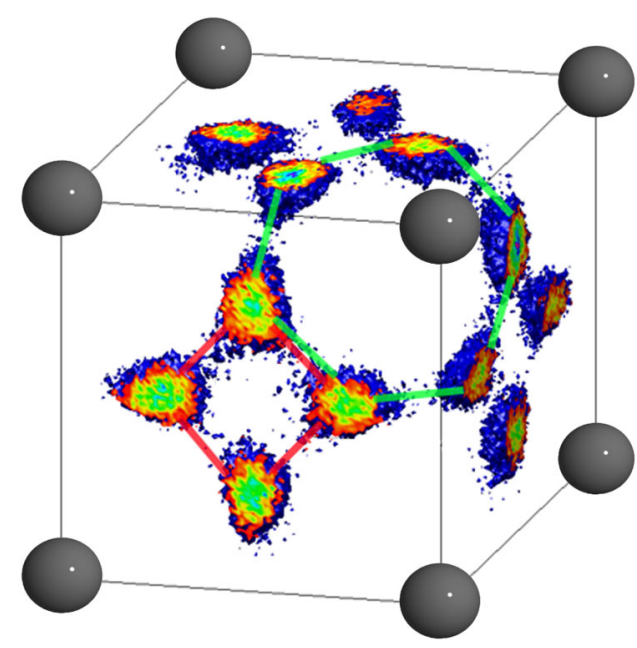

FIG. 2 (color online). An isosurface of the hydrogen density in bcc $\mathrm{Nb}$ at $550 \mathrm{~K}$. Notice how the points cluster around the tetrahedral sites in excellent agreement with experimental results [2]. The lines connecting the tetrahedral sites show the $4 T$ and $6 T$ rings. The gray spheres are $\mathrm{Nb}$ atoms.
The energy landscape of a hydrogen atom self-trapped at a tetrahedral site is illustrated in Fig. 1. The potential far away from the hydrogen atom, describing the potential in the absence of self-trapping, is shown in the upper left. The energy of the surrounding sites can be obtained at $0 \mathrm{~K}$, by moving the proton to different sites while keeping the lattice, including the self-trapped state, frozen. Tracing the energy of the $T$ sites yields the potential seen by instantaneous hopping of hydrogen and we define the resulting energy landscape as the excited states of the quasiparticle. The filled point symbolizes the proton in its self-trapped state, and the corresponding self-trapping energy is denoted by $E_{S}$. Three cases are considered in the lower panel of Fig. 1: a jump from the self-trapped site to one of the nearest tetrahedral sites on the $4 T$ ring resulting in an energy difference $E_{\mathrm{I}}=0.071 \mathrm{eV}$, a double jump process on the $4 T$ ring, denoted as IIa with the energy $E_{\mathrm{IIa}}=0.148 \mathrm{eV}$, and a double jump on the $6 T$ ring, giving $E_{\mathrm{IIb}}=0.189 \mathrm{eV}$ is denoted as IIb. These energies describe the energy landscape within the polaron, and should not be confused with activation energies for hydrogen diffusion, since the energies correspond to differences between energy levels, not barrier heights, and the potential is dynamic. The equilibrium self-trapped energy at $0 \mathrm{~K}$ was determined to be $E_{S}=0.206 \mathrm{eV}$ including zero point energy (ZPE) and $0.170 \mathrm{eV}$ without $\mathrm{ZPE}$, which is in agreement with the results of Sundell and Wahnström $(0.189 \mathrm{eV})[8,9]$.

The diffusion coefficients were calculated from the mean residence time of hydrogen, $\langle\tau\rangle$, [19] in the temperature range 400 to $2400 \mathrm{~K}$ and are plotted in Fig. 3.

The activation energies in regions II and III are 0.151(4) and $0.176(5) \mathrm{eV}$, respectively. No experimental results are available for temperatures corresponding to region III in

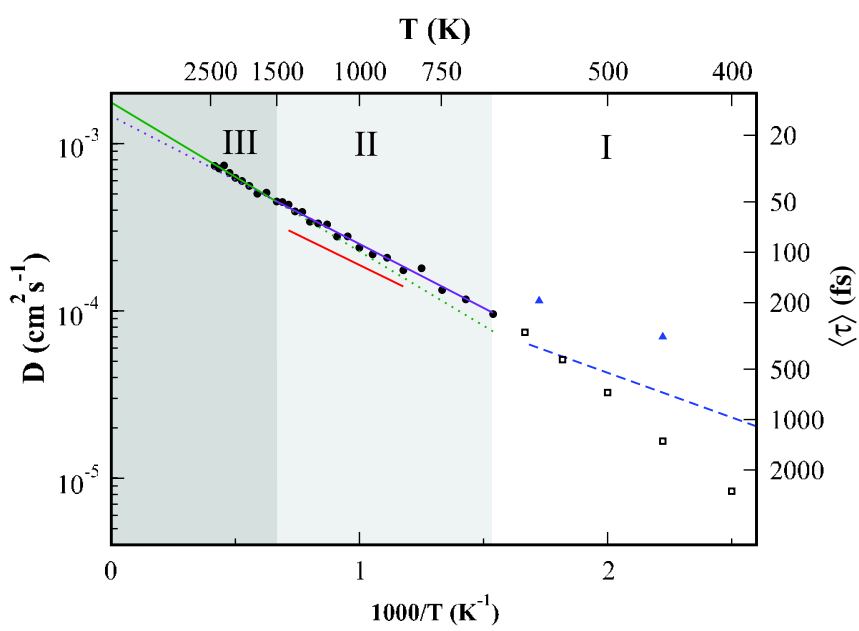

FIG. 3 (color online). Arrhenius plot of the calculated and experimental diffusion coefficients. The black circles and squares show our calculated diffusion rates. The experimentally obtained rates are drawn in red (solid, 873-1390 K) [20] and blue (dashed) [26]. The blue triangles are results obtained from classical MD [23]. 
the figure, while the results in region II are in excellent agreement with the experimental data, 0.144(3) eV [20]. In region I, the agreement is less compelling. The limited cell size will cause an artificial polaron self-interaction, through the periodic boundary conditions. Using the cell size $(10 \AA)$ and the speed of sound in $\mathrm{Nb}(34 \AA / \mathrm{ps})$ we determined the characteristic interaction time to be $300 \mathrm{fs}$, which agrees well with $600 \mathrm{~K}$ as a crossing point. We cannot, however, rule out tunneling and ZPE effects, which are not included.

Now let us consider the effect of self-trapping on the local strain of the niobium lattice. We have used the first peak of the $\mathrm{H}-\mathrm{Nb}$ radial distribution function as a measure of the amplitude of the local strain field ( $\mathrm{H}-\mathrm{Nb}$ distance) in the immediate vicinity of the interstitial. In the hydrogenfree niobium lattice, the distance from the interstitial tetrahedral site to the four closest $\mathrm{Nb}$ atoms was determined to be $1.86 \AA$ at $0 \mathrm{~K}$. By plotting (Fig. 4) this distance as a function of temperature we can follow the changes in the amplitude of the local strain field. The results displayed in Fig. 4 are obtained from simulations performed at a constant volume. Ignoring the thermal expansion eased the calculations and the extraction of the hydrogen induces changes in the atomic distances. This approach is motivated by the negligible influence of the volume on the difference in the $\mathrm{H}-\mathrm{Nb}$ distances at different residence times, which was verified by 900 and $1500 \mathrm{~K}$ simulations with different volumes of the cell. As seen in the figure, the average distance exhibits a close to linear decrease with increasing temperature to approximately $1000 \mathrm{~K}$ where it levels out. It is also worth noting that the distance does not reach $1.86 \AA$, which is the distance in the hydrogen-free lattice. Thus, a local strain field is always

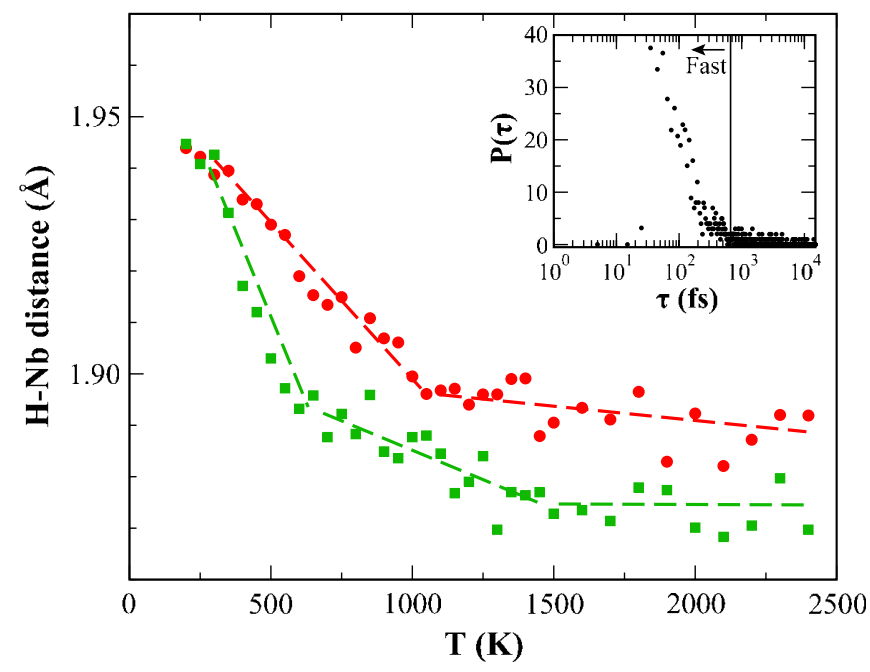

FIG. 4 (color online). The average distance between hydrogen and the nearest neighbor $\mathrm{Nb}$ as a function of temperature (red circles). The probability of residence, $P(\tau)$, as a function of residence time $(\tau)$, at $500 \mathrm{~K}$, is illustrated as an inset. The green squares show the $\mathrm{H}-\mathrm{Nb}$ distance for residence times smaller than the average residence time, $\langle\tau\rangle$. present, while its amplitude and distribution depends on the temperature.

In 1986 Dosch and Peisl [21,22] measured the hydrogen diffusion in $\mathrm{Nb}$ from room temperature to $573 \mathrm{~K}$ using quasielastic neutron scattering. They saw an oscillatory dependence of the structure factor on the momentum transfer, which was interpreted assuming the existence of a so-called mobile state of hydrogen. This mobile state corresponds to a nonequilibrium state where the local distortion does not have time to fully relax over a sequence of rapid jumps (10-100 fs) between neighboring tetrahedral sites. Wahnström and Li supported this idea in 1993 using classical molecular dynamics at 450 and $580 \mathrm{~K}$ [23]. Dosch et al. [24] argued that the nonequilibrium state could be the reason for the cubic symmetry of the local strain field measured experimentally, even though the tetrahedral site itself has a tetragonal symmetry. However, in 1994 Elsässer et al. [25] showed by electronic structure calculations that a hydrogen at a tetrahedral site produces an isotropic strain field. Static forces are thus sufficient to reproduce the cubic symmetry of the strain field and consequently there is no need to invoke a dynamic force field. We have confirmed this finding from our $0 \mathrm{~K}$ calculations.

Wahnström and Li [23] found from their analysis of the jump angles at $450 \mathrm{~K}$ that the proportion of jumps back to the last occupied $T$ site is much larger in comparison to what would be expected, which we have confirmed. The overrepresentation of jumps back to the last occupied $T$ site can be understood from the difference in energy levels as shown in Fig. 1. Immediately after the jump between $T$ sites, the polaron is in an excited state. The deexcitation has two branches, return to the initial $T$ site, or a translation of the local strain field. The first excitation of the polaron, $E^{\prime}$, has an excitation energy in the range $0<E_{1}^{\prime}<E_{\mathrm{I}}$, see Fig. 1. A double jump corresponds to a higher excitation, with an energy in the range $0<E_{2}^{\prime}<E_{\mathrm{IIb}}$, depending on the direction and the time required for the double jump. The probability of higher excitations increases with increasing temperature. The upper limit for the excitation of the polaron is defined by the self-trapping energy of the hydrogen. The internal excitation of the polaron results in an increased activation energy, which is consistent with experiments as well as our findings.

The increase of the hopping rate with increasing temperature, combined with the temperature independent relaxation time of the lattice, results in an unbinding of the hydrogen strain field pair. As a consequence, the amplitude of the local lattice displacement must depend on $\tau$. To prove this point, we plot, in Fig. 4, the $\mathrm{H}-\mathrm{Nb}$ distance obtained for $\tau \leq\langle\tau\rangle$, as a function of temperature. The choice of time scale is somewhat arbitrary, but serves the purpose of separating the long and short residence times. As seen from Fig. 4, the mean displacement, and the displacement developed around the fastest moving hydrogen, is the same at and below room temperature. Thus, at these temperatures, the proton and the strain field 


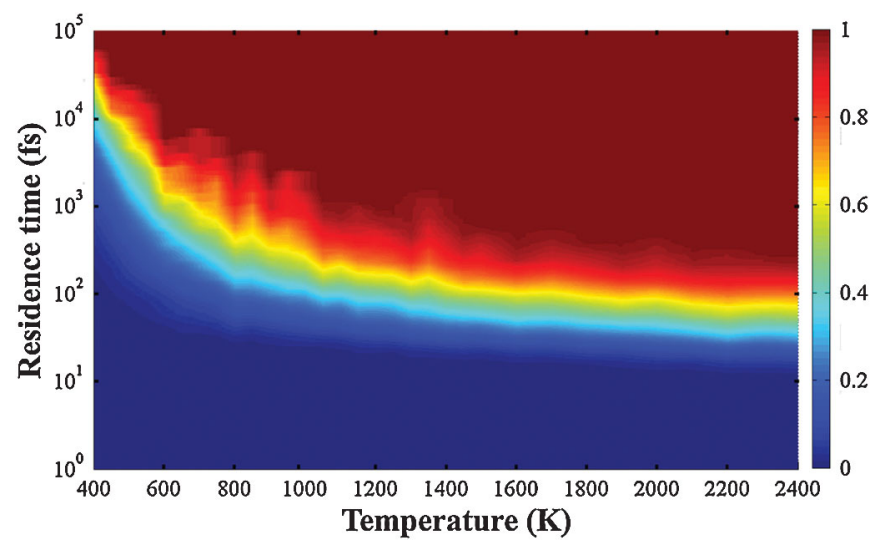

FIG. 5 (color). Two-dimensional mapping of the jump probability as a function of residence time and temperature, showing the probability that the proton has jumped to the next tetrahedral site.

are fully coupled and diffuse as a unity (polaron diffusion). Above room temperature, we can see a difference in the strain field amplitude depending on whether the protons have long or short residence times.

Above $1500 \mathrm{~K}$ the change in displacement with temperature is weak, which we interpret as the limit of the unbinding between the proton and the strain field. Therefore, this temperature serves as a natural dividing line between regions II and III in Fig. 3. A distinct difference in the activation energies is seen between these two regions, 0.151(4) $\mathrm{eV}$ in region II and $0.176(5) \mathrm{eV}$ in region III. We note that a partial self-trapped state is still found at the highest temperatures, as the displacement is not the same for all $\tau$. Thus, the unbinding of the polaron is not complete, even at the highest temperatures. We did not detect any stress dependence on the proton residence time, from an analysis of the cell stress. This implies that even though the local strain field around the proton diminishes, the global strain field does not; i.e., there must be a strain field trail following the diffusing hydrogen.

From the $\mathrm{H}-\mathrm{Nb}$ radial distribution it was clear that the probability of self-trapping decreases rapidly with increasing temperature. The same effect can be observed by looking at the hydrogen jump probability. As seen in Fig. 5, the residence times become shorter as the temperature is increased, for the same probability of executing a successful jump. Or conversely, fast moving hydrogen atoms are more likely to execute successful jumps as the temperature is increased. This is just another way of saying that the probability for a hydrogen atom to become selftrapped decreases with increasing temperature.

In conclusion, we have demonstrated a temperature dependent unbinding of the proton and its local strain field. At elevated temperatures this effect results in a strain field trail, reflecting the diffusion trace of the interstitial.

When reinterpreting the experimental results $[20-22,24]$, on the basis of our findings, the importance of the relaxation time of the lattice on the hydrogen diffusion becomes apparent. Internal excitations within the polaron are a consequence of the short time constants of the dynamics of the light interstitial, as compared to the relaxation time of the lattice. This will influence the $\mathrm{H}-\mathrm{H}$ interaction and is expected to be of great importance for understanding the collective dynamic behavior, such as order-disorder transitions, in all transition metal-hydrogen systems.

The authors would like to thank the Nordic Energy Research Program (NERP) and the Swedish research councils, VR and Formas, for financial support. Computer time was provided by SNIC and UPPMAX.

[1] G. Alefeld and J. Vökl, Hydrogen in Metals I (SpringerVerlag, Berlin, 1978).

[2] G. Alefeld and J. Vökl, Hydrogen in Metals II (SpringerVerlag, Berlin, 1978).

[3] H. Wipf, Hydrogen in Metals III (Springer-Verlag, Berlin, 1997).

[4] Y. Fukai, The Metal-Hydrogen System: Basic Bulk Properties (Springer-Verlag, Berlin, 2005).

[5] Y. Fukai, Jpn. J. Appl. Phys. 22, 207 (1983).

[6] P. H. Andersson, L. Fast, L. Nordström, B. Johansson, and O. Eriksson, Phys. Rev. B 58, 5230 (1998).

[7] Y. G. Li and G. Wahnström, Phys. Rev. B 51, 12233 (1995).

[8] P. G. Sundell and G. Wahnström, Phys. Rev. Lett. 92, 155901 (2004).

[9] P. G. Sundell and G. Wahnström, Phys. Rev. B 70, 224301 (2004).

[10] C. P. Flynn and A. M. Stoneham, Phys. Rev. B 1, 3966 (1970).

[11] R. Hempelmann, J. Less-Common Met. 101, 69 (1984).

[12] H. R. Schober and A. M. Stoneham, Phys. Rev. Lett. 60, 2307 (1988).

[13] A. M. Stoneham, J. Gavartin, A. L. Shluger, A. V. Kimmel, D. M. Ramo, H. M. Ronnow, G. Aeppli, and C. Renner, J. Phys. Condens. Matter 19, 255208 (2007).

[14] W. Kohn and L. J. Sham, Phys. Rev. 140, A1133 (1965).

[15] G. Kresse and J. Furthmüller, Phys. Rev. B 54, 11169 (1996).

[16] G. Kresse and D. Joubert, Phys. Rev. B 59, 1758 (1999).

[17] J. P. Perdew and Y. Wang, Phys. Rev. B 45, 13244 (1992).

[18] P. E. Blöchl, Phys. Rev. B 50, 17953 (1994).

[19] J. M. Rowe, K. Sköld, H. E. Flotow, and J. J. Rush, J. Phys. Chem. Solids 32, 41 (1971).

[20] T. Eguchi and S. Morozumi, J. Jpn. Inst. Met. 41, 795 (1977).

[21] H. Dosch and J. Peisl, Phys. Rev. Lett. 56, 1385 (1986).

[22] H. Dosch, J. Peisl, and B. Dorner, Phys. Rev. B 35, 3069 (1987).

[23] G. Wahnström and Y.G. Li, Phys. Rev. Lett. 71, 1031 (1993).

[24] H. Dosch, F. Schmid, P. Wiethoff, and J. Peisl, Phys. Rev. B 46, 55 (1992).

[25] C. Elsässer, M. Fähnle, L. Schimmele, C. T. Chan, and K. M. Ho, Phys. Rev. B 50, 5155 (1994).

[26] Z. Qi, J. Völkl, R. Lässer, and H. Wenzl, J. Phys. F 13, 2053 (1983). 